\title{
El costo de capital y su incidencia en los métodos de flujo de caja descontados
}

\author{
J. R. Medina* y G. A. Pérez \\ Cátedra de Proyecto Industrial e Ingeniería Económica \\ Facultad de Ingeniería Química (Universidad Nacional del Litoral), \\ Santiago del Estero 2829, S3002 GJC Santa Fe, R Argentina. \\ jrmedina@fiq.unl.edu.ar
}

(Recibido/received: 28-Febrero-2012; aceptado/accepted: 30-Junio-2012)

\section{RESUMEN}

Un estudio de factibilidad técnico económico en cualquier rama de la ingeniería o en cualquier tipo de negocio, implica conocer una medida de la rentabilidad del emprendimiento. Actualmente, nadie discute que para una toma de decisión fundamentada se deben utilizar los métodos de flujo de caja descontados (DCF), a pesar de que persisten otras formas muy difundidas (por ejemplo: período de repago, relación beneficio/costo). El objetivo del trabajo es analizar críticamente los métodos a utilizar para evaluar proyectos mutuamente excluyentes, como también proponer formas alternativas de análisis de rentabilidad que contemplen la incidencia de las distintas tasas y acoten la decisión de un modo adecuado. La propuesta presentada aquí permite ordenar alternativas excluyentes con criterios que contengan una visión mas completa. El resultado más interesante está relacionado con la sensibilidad de los métodos sobre las variables de decisión inherentes a estos.

Palabras clave: DCF, costo de capital; proyectos excluyentes; rentabilidad de proyectos.

\begin{abstract}
A techno-economic feasibility study in any branch of engineering or any kind of business involves knowing a measure of the investment profitability. Currently, no one discuss that for a solid basis decision making it should be used discounted cash flow (DCF), despite the persistence of a widespread forms (i.e. payback period, benefit / cost). The objective of this paper is to analyze, critically, the relevance of the methods used to make comparisons between mutually exclusive projects as well as suggest alternative ways of analysis that consider the impact of different rates of capital cost and constrain the decision in an appropriate way. The proposal presented here allows exclusive alternatives ordering with an approach that include a more complete view on the problem. The most interesting result is related to the sensitivity of the methods on the inherent decision variables involved in them.
\end{abstract}

Keywords: DCF; cost of capital; mutually exclusive alternatives; project profitability

\footnotetext{
* Autor para la correspondencia
} 
Medina, J. y Pérez, G.

\section{INTRODUCCIÓN}

Si los criterios se apoyan en los denominados DCF, sin excepción, lo que motiva discusión (Liu et all, 2004) es la pertinencia de cual usar para establecer comparaciones entre proyectos mutuamente excluyentes o el significado de la tasa de corte o del retorno mínimo aceptable. Esto es porque los modelos discretos, axiomáticamente, se basan en la Equivalencia de Dinero, por lo que la tasa de descuento se transforma en una variable clave del problema. En consecuencia, el más usado método del Valor Actual o Presente Neto (VAN) es un funcional cuyo resultado es debido a los valores de los flujos de caja de todos los períodos (incluida la inversión total inicial), el horizonte de planificación y el denominado costo de capital. Y, los que se derivan de él implican la misma consideración. Asimismo, los métodos de la Tasa Interna de Retorno (TIR) Modificados reiteran la dependencia en la decisión con el valor de la tasa de descuento, aplicada y/o usada como comparación (tasa atractiva mínima de retorno).

\section{ENUNCIADOS Y ANÁLISIS.}

Habida cuenta de la necesidad de contabilizar el valor del dinero en el tiempo surgen los factores de capitalización y de descuento, ya sea definidos siguiendo un modelo discreto o uno continuo, que se aplican para poder comparar las sumas presentes y futuras. Pero, en realidad, el concepto fundamental para toda consideración posterior es el teorema de Equivalencia de Dinero, Esta idea es esencial porque la tasa elegida garantiza la condición necesaria y suficiente del mencionado teorema. Y, pone de manifiesto el concepto de variable de decisión independiente, con gran incidencia en el resultado buscado.

Se puede definir un funcional que contenga todas las variables que participan en la elección de una alternativa de inversión, caracterizando a cada una de ellas. La vida del emprendimiento (de la inversión), del equipamiento, denominada comúnmente horizonte de planificación, también, es una variable independiente en el análisis.

El énfasis, en los métodos que contemplan la incertidumbre y el riesgo, se pone en los flujos de caja de los diferentes años, causa esencial de la variabilidad, dejando las otras variables como determinísticas (Park \& Sharp-Bette, 1990). Si bien es cierto que los escenarios previstos se reflejan en los flujos de fondos que resultan, esta no es la única razón que puede hacer fracasar una idea. Por lo tanto, la "receta" del Valor
Esperado del Valor Presente Neto debe estar acompañada por algunos conceptos que fortalezcan la toma de decisión.

Como se tienen valores en distintos tiempos, es fácil generalizar una función (funcional, en realidad), que se defina en un punto determinado cualquiera del horizonte de planificación, a pesar de que lo usual es el inicio (valor presente) o el fin de la vida del proyecto. Esto es,

$$
V(j)=V\left[F_{c i}, I_{T 0}, k, N\right] ; i=1, \ldots N
$$

Donde se expresan todos los flujos de caja (a partir del primer año), la inversión total inicial, el costo del capital y el horizonte de planificación como las variables de la función básica de todo método de flujo de caja descontado. Siendo j cualquier año del análisis y el resultado un número real.

A partir de este enunciado se puede demostrar que ese número es una consecuencia de todos los valores implicados. Y que cualquier estudio de sensibilidad completo debe analizar el comportamiento del funcional frente a la incertidumbre en todas las variables de decisión.

Hay un trabajo reciente (Borgonovo, E, Peccati, L, 2004) que puntualiza el problema y propone un análisis de esas variaciones $y$, también, una propuesta anterior que trabaja el problema con un modelo simplificado (Pérez G, 2002).

Si a la ecuación (1) la expresamos en forma genérica es:

$$
V(j)=\sum_{i=1}^{j} F_{c i}(1+k)^{j-i}+\sum_{i=j+1}^{N} \frac{F_{c i}}{(1+k)^{i-j}}-I_{T 0}(1+k)^{j}
$$

Donde se ven todas las variables que conducen a la obtención de ese número, que debe ser mayor que cero para que el emprendimiento sea factible. Tomando el caso usual del Valor Actual (o Presente), para la discusión, es:

$$
V A N=\sum_{i=1}^{N} \frac{F_{c i}}{(1+k)^{i}}-I_{T 0}
$$

Y, comparando dos proyectos,

$$
V A N_{I}-V A N_{I I}=\sum_{i=1}^{N} \frac{\left(F_{c l i}-F_{c l l i}\right)}{(1+k)^{i}}-\left(I_{T 0 I}-I_{T 0 I I}\right)
$$

Vol. 25, No. 01, pp. 18-21/Junio 2012 
Medina, J. y Pérez, G.

Simplificando, para ver la incidencia de las variables en forma más clara, sobre todo en el conjunto de todas las variables

$$
V A N_{I}-V A N_{I I}=\left(F_{c l}-F_{c l}\right)\left[\frac{(1+k)^{N}-1}{(1+k)^{N} k}\right]-\left(I_{T 0 I}-I_{T O I}\right)
$$

Donde hemos quitado la variabilidad a los flujos de fondos, tomando un valor constante promedio para todo el horizonte de planificación, y así analizar los otros elementos, con mayor énfasis. Si se consideran valores del costo de capital razonable, esto es valores bajos de k, se puede hacer la siguiente aproximación:

$$
\left[\frac{(1+k)^{N}-1}{(1+k)^{N} k}\right] \cong \frac{N}{(1+N k)}
$$

Aplicándola en la ecuación (5), muestra que si (en valores constantes o promedios) los flujos de caja son suficientemente mayores y el $\mathrm{N}$ es grande, podría ser atractiva una alternativa aún con una inversión inicial mucho mayor. Que es coherente con la hipótesis de costo de dinero "barato", expresión comúnmente escuchada.

$\mathrm{Si}$, por otra parte, interesa analizar la influencia del costo del dinero individualmente se puede suponer un cálculo del VAN con un valor del mismo "perturbado", afectándolo por un infinitésimo. Es decir,

$$
V A N=\sum_{i=1}^{N} \frac{F_{c i}}{(1+k)^{i}}-I_{T O}
$$

Y, el modificado como

$V A N^{*}=\sum_{i=1}^{N} \frac{F_{c i}}{(1+k+\varepsilon)^{i}}-I_{T 0}$

Utilizando una aproximación, como la siguiente, es

$$
(1+k+\varepsilon)^{i} \cong(1+k)^{i}\left[1+\frac{\varepsilon i}{(1+k)}\right]
$$

Resulta, haciendo la comparación,

$$
V A N-V A N^{*} \cong \sum_{i=1}^{N} \frac{F_{c i}}{(1+k)^{i}}\left[\frac{\varepsilon i}{(1+k)+\varepsilon i}\right]
$$

Donde se demuestra que el VAN sin alteraciones será mayor al perturbado, para cualquier valor de la perturbación mayor a cero.

En definitiva, el costo del capital incide directamente sobre el resultado, aún con pequeñas variaciones del mismo.

Y, tomando solamente la ecuación perturbada es muy ilustrativa porque resulta

$$
V A N^{*} \cong \sum_{i=1}^{N} \frac{F_{c i}}{(1+k)^{i}}\left[\frac{1+k}{1+k+\varepsilon i}\right]-I_{T 0}
$$

Que muestra como disminuyen, término a término, los flujos descontados para cualquier valor de la perturbación.

Retomando la aproximación de la ecuación (6), se puede demostrar (Pérez, G, 2003) que para que el emprendimiento sea factible (Valor Actual o Presente Neto mayor que cero), debe ser:

$$
\frac{N}{(1+N k)} \geq t_{R}
$$

Donde $t_{R}$ representa el período de repago, en su definición clásica. Reafirmándose la importancia de las variables horizonte de planificación y costo de capital, como se ha argumentado a lo largo del trabajo. Ya que la ecuación (11) muestra un criterio simple que relaciona valores que la experiencia y/o la intuición de los que toman las decisiones conocen.

\section{CONCLUSIÓN}

La receta de elegir una inversión si el VAN o la TIR "aprueban el examen", sin considerar ningún otro factor, es insuficiente en la toma de decisiones económicasfinancieras. Se ha tratado, a lo largo de este análisis, enfatizar el hecho que estos métodos se basan en un funcional; en donde el valor y la variabilidad del costo del dinero, fundamentalmente, y la vida del proyecto inclusive, juegan un rol que puede ser preponderante. 


\section{REFERENCIAS.}

Borgonovo E, Peccati L, "Sensitivity analysis in investment project evaluation", Int. J. Production Economics, Volume 90, Issue 1, Pages 17-25. (2004). Eschenbach Teg G. "Engineering Economy. Applying theory to practice". Ed. Irwin (1995)

Liu, L, A Rettenmaier, T Saving. "Present Value Criterion: the case of differing borrowing and lending rates", Economics Letters, Vol. 84, Issue 3. Pages 427-432. (2004)

Park C S, Sharp-Bette G P, Advanced Engineering Economics, J Wiley \&Sons (1990)

Pérez G. "Métodos de Evaluación de Rentabilidad. Una Revisión Crítica". Información Tecnológica, Vol. 13, No 2, pag. 159-163 (2002)

Pérez G. "Preliminary Economic Decisions. A Simplified Approach", Cost Engineering, Vol 45, N 10, pag. 30-32 (2003)

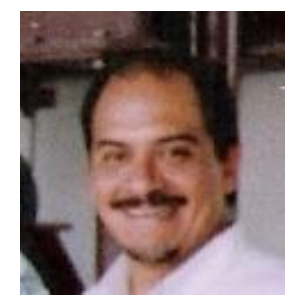

José R. Medina es Ingeniero Químico y Magister en Ciencia y Tecnología de Alimentos, recibido en la Universidad Nacional de Litoral (Argentina). Participa como Profesor de asignaturas de Ingeniería Económica y Proyecto Industrial, y con tareas de investigación sobre Tecnologías de procesamiento y conservación de matrices cárnicas.

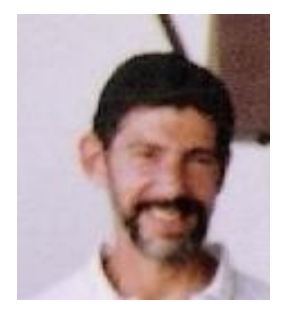

Gustavo A. Pérez es Ingeniero Químico, recibido en la Universidad Nacional del Litoral (Argentina). Desarrolla sus actividades como Profesor Responsable de las asignaturas Ingeniería Económica y Proyecto Industrial en la Facultad de Ingeniería Química. Además realiza tareas de investigación en el CONICET, en el área de "Ingeniería de Procesos" 